\title{
WEIGHTED INEQUALITIES THROUGH FACTORIZATION
}

\author{
EUGENIO HERNÁNDEZ
}

\section{Introduction and results}

In [4] $P$. Jones solved the question posed by $B$. Muckenhoupt in [7] concerning the factorization of $A_{p}$ weights. We recall that a non-negative measurable function $w$ on $\mathbf{R}^{n}$ is in the class $A_{p}, 1<p<\infty$ if and only if the Hardy-Littlewood maximal operator is bounded on $L^{p}\left(\mathbf{R}^{n}, w\right)$. In what follows $L^{p}(X, w)$ denotes the class of all measurable functions $f$ defined on $X$ for which $\left\|f w^{1 / p}\right\|_{L p(X)}<\infty$, where $X$ is a measure space and $w$ is a non-negative measurable function on $X$.

It has recently been proved that the factorization of $A_{p}$ weights is a particular case of a general factorization theorem concerning positive sublincar operators. The case in which the operator is bounded from $L^{p}(X, v)$ to $L^{p}(Y, u), 1<p<$ $\infty$, for $u$ and $v$ non-negative measurable functions on $X$ and $Y$ respectively, is treated in [8]. The case in which the operator is bounded from $L^{p}(X, v)$ to $L^{q}(X, u), 1<p<q<\infty$ is treated in [3].

Our first result is a factorization theorem for weights $u$ and $v$ associated to operators bounded from $L^{p}(X, v)$ to $L^{q}(Y, u)$, where $X$ and $Y$ are two, possibly different, measure spaces, and $p$ and $q$ are any index between 1 and $\infty$.

Let $X$ and $Y$ be two measure spaces and let $M(X), M(Y)$ be the class of measurable functions defined in $X$ and $Y$ respectively. An operator $T$ defined on a subset of $M(X)$ with values in $M(Y)$ is called sublinear if $|T(f+g)| \leq$ $|T(f)|+|T(g)|$ and is called positive if $|f| \leq g \mapsto|T(f)| \leq T(g)$, for all $f, g \in M(X)$ which belong to the domain of $T$.

Theorem I (Factorization). Let $T$ and $T$ be two positive sublinear operators defined on subsets of $M(X)$ and $M(Y)$ respectively. Let $v \in M(X)$ and $u \in M(Y)$ be non-negative functions and $1<p, q<\infty$. Suppose that $T$ is bounded from $L^{p}(X, v)$ to $L^{q}(Y, u)$ with norm $\|T\|$ and $T^{\prime}$ is bounded from $L^{q^{\prime}}\left(Y, u^{-q^{\prime} / q}\right)$ to $L^{p^{\prime}}\left(X, v^{-p^{\prime} / p}\right)$ with norm $\left\|T^{\prime}\right\|$. Then there exist non-negative functions $u_{0} \in M(X), v_{0} \in M(Y), u_{1} \in M(Y)$ and $v_{1} \in M(X)$ such that $v=u_{0}^{-p / p^{\prime}} v_{1}, u=v_{0}^{-q / q^{\prime}} u_{1},\left\|u_{0} v_{1}\right\|_{L^{\prime}(X)} \leq 1,\left\|v_{0} u_{1}\right\|_{L^{2}(Y)} \leq 1, T\left(u_{0}\right) \leq\|T\| v_{0}$ and $T^{\prime}\left(u_{1}\right) \leq 2^{p / p^{\prime}}\left\|T^{\prime}\right\| v_{1}$.

This theorem can be applied to a large class of operators to obtain the factorization of their associated weights. The reader can find several examples in $[8]$ and [3]. 
For integral operators with non-negative kernel, the factorization theorem has a converse for some particular cases of $p$ and $q$. Let $k(x, y)$ be a measurable nonnegative function on $X \times Y$. Let us denote by $K$ and $K^{*}$ the transformations:

$$
(K f)(y)=\int_{X} k(x, y) f(x) d x, \quad\left(K^{*} g\right)(x)=\int_{Y} k(x, y) g(y) d y,
$$

the domain of $K$ being the set of all functions $f \in M(X)$ such that the first integral exists and is finite for almost all $y$, and the domain of $K^{*}$ being analogously defined.

Theorem 2. Let $1<q<p<\infty$ and $v \in M(X), u \in M(Y)$ be non-negative. $A$ necessary and sufficient condition for $K$ to be bounded from $L^{p}(X, v)$ to $L^{Q}(Y, u)$ is that there exist non-negative functions $u_{0} \in M(X), v_{0} \in M(Y), u_{1} \in$ $M(Y), v_{1} \in M(X)$ and finite constants $C_{0}, C_{1}$ such that $\left\|u_{0} v_{1}\right\|_{L^{1}(X)} \leq 1, v=$ $u_{0}^{-p / p^{\prime}} v_{1}, u=v_{0}^{-q / q^{\prime}} u_{1}, K\left(u_{0}\right) \leq C_{0} v_{0}$ and $K^{*}\left(u_{1}\right) \leq C_{1} v_{1}$. Moreover $\|K\| \leq$ $C_{0}^{1 / q^{\prime}} C_{1}^{1 / 9}$.

The case $p=q$ is simpler:

Theorem 3. Let $v \in M(X)$ and $u \in M(Y)$ be non-negative. A necessary and sufficient condition for $K$ to be bounded from $L^{p}(X, v)$ to $L^{p}(Y, u)$ is that there exist non-negative functions $u_{0} \in M(X), v_{0} \in M(Y), u_{1} \in M(Y), v_{1} \in$ $M(X)$ and finite constants $C_{0}, C_{1}$ such that $v=u_{0}^{-p / p^{\prime}} v_{1}, u=v_{0}^{-p / p^{\prime}} u_{1}, K\left(u_{0}\right)$ $\leq C_{0} v_{0}$ and $K^{*}\left(u_{1}\right) \leq C_{1} v_{1}$. Moreover $\|K\| \leq C_{0}^{1 / p^{\prime}} C_{1}^{1 / p}$.

The case $v \equiv u \equiv 1$ of theorems 2 and 3 is proved in [1]. Our proof of these theorems is an adaptation of the proof of the corresponding results in [1]. In the case $p<q$ the conditions of theorem 2 are not sufficient for the boundedness of $K$ from $L^{p}(X, v)$ to $L^{q}(X, u)$ even in the case $v \equiv u \equiv 1$ (see [1]). Observe that in theorem 2 we only need the condition $\left\|u_{0} v_{1}\right\|_{L^{2}(X)} \leq 1$ while the "symmetric" condition $\left\|u_{1} v_{0}\right\|_{L^{\prime}(Y)} \leq 1$ is not needed. Neither of these is needed in theorem 3 .

For some applications it is better to replace the sufficient condition of theorem 2 by the following one, whose statement is a generalization of the sufficient condition of theorem 3 :

Theorem 4. Let $1<q<p<\infty$ and $v \in M(X), u \in M(Y)$ be non-negative. Suppose that there exist non-negative mesurable functions $u_{0}, v_{0}, u_{1}, v_{1}$ such that $v=u_{0}-p / p^{\prime} v_{1} q^{\prime} / p^{\prime}, u=u_{1} v_{0}-p / q^{\prime}, K\left(u_{0}\right) v_{0}^{-1} \in L^{r}(u)$ (with $L^{r}(u)$ norm equal to $C_{0}$ ) and $K^{*}\left(u_{1}\right) v_{1}^{-1} \in L^{r}\left(v^{-p^{\prime} / p}\right)$ (with $L^{\top}\left(v^{-p^{\prime} / p}\right)$ norm equal to $C_{1}$ ), where $\frac{1}{r}=\frac{1}{q}-\frac{1}{p}$. Then, $K$ is a bounded operator from $L^{p}(X, v)$ to $L^{q}(Y, u)$ with norm less then or equal to $C_{0} r^{\prime} / \Phi^{\prime} C_{1}^{r^{\prime} / p}$.

For the cases $q=1$ or $p=\infty$, which are not covered by the above theorems, we have the following satisfactory result: 
Theorem 5. (A) If $1 \leq p \leq \infty$, a necessary and sufficient condition for $K$ to be bounded from $L^{p}(X, v)$ to $L^{1}(Y, u)$ with norm $\|K\|$ is

$$
\left\|\int_{Y} k(x, y) u(y) d y\right\|_{L \rho^{\prime}\left(X, y^{\left.-s^{\prime} / p\right)}\right.} \leq\|K\|
$$

(B) If $1 \leq q \leq \infty$, a necessary and sufficient condition for $K$ to be bounded from $\underline{L}^{\infty}(X, v)$ to $L^{q}(Y, u)$ with norm $\|K\|$ is

$$
\left\|\int_{X} k(x, y) v^{-1}(y) d y\right\|_{L q}(Y, u) \leq\|K\|
$$

In this theorem $\underline{L}^{\infty}(X, v)=\left\{f \in M(X):\|f v\|_{\infty}<\infty\right\}$.

Examples of operators to which these theorems can be applied are the following: the Hardy operator

$$
T f(x)=\int_{0}^{x} f(y) d y \quad, x>0
$$

and its duat

$$
T^{*} f(x)=\int_{x}^{\infty} f(y) d y \quad, x>0
$$

the fractional integral operator

$$
\left(I_{\alpha} f\right)(x)=\int_{\mathbf{R}^{n}} f(x-y)|y|^{\alpha-n} d y, \quad x \in \mathbf{R}^{n}, 0 \leq \alpha<n
$$

which is self-adjoint; the Riemann-Liouville operator

$$
\left(T_{\alpha} f\right)(x)=\frac{1}{\Gamma(\alpha)} \int_{0}^{x} \frac{f(y)}{(x-y)^{1-\alpha}} d y, \quad x \geq 0, \alpha \geq 0
$$

the Laplace transform

$$
\mathcal{L} f(x)=\int_{0}^{\infty} e^{-x y} f(y) d y
$$

and the multidimensional Hardy operator

$$
T_{n} f\left(x_{1}, \ldots, x_{n}\right)=\int_{0}^{x_{1}} \cdots \int_{0}^{x_{n}} f\left(y_{1}, \ldots, y_{n}\right) d y_{n} \ldots d y_{1} .
$$

The proofs of theorems 1 to 5 will be given in section 2. Applications will be given in section 3 . These are concerned with weighted inequalities for some of the above operators.

I watd like to thank $B$. Jawerth for calling my attention to [1], which turned out to be the starting point of this research. 


\section{Proofs of Theorems 1 to 5}

To prove theorem 1 we need the following lemma which can be found in [1].

Lemma 2.1. Let $B$ be a Banach space and $P$ a convex cone in $B$. By calling this cone "positive", $B$ will be taken as an ordered Banach space. Let us suppose for $B$ and $P$ that every bounded increasing sequence in $P$ converges, more precisely:

$$
\left\{f_{n}\right\} \subset P, f_{n+1}-f_{n} \in P,\left\|f_{n}\right\| \leq M<\infty \Rightarrow f_{n} \rightarrow f \in P
$$

Let $S$ be a transformation defined in $B$ such that $S(P) \subset P, S$ is nondecreasing (that is, $f, g, g-f \in P \Rightarrow S g-S f \in P$ ), $S$ is continuous and $\|f\| \leq 1 \Rightarrow$ $\|S f\| \leq C_{0}<\infty$.

Then there exists $\alpha \in P, \alpha \neq 0,\|\alpha\| \leq 1$ such that

$$
2 C_{0} \alpha-S \alpha \in P
$$

To prove theorem 1 we take $B=L^{p}(X), P$ such that $f \in P \Leftrightarrow f(x) \geq 0$ o.e. and

$$
S(f)=\left[T^{\prime}\left(\left(\frac{T\left(f v^{-1 / p}\right)}{\|T\|}\right)^{q / q^{\prime}} u\right)\right]^{p^{\prime} / p} v^{-p^{\prime} / p^{2}}
$$

and apply lemma 2.1. Observe that the boundedness of $T$ and $T^{\prime}$ implies

$$
\|S f\|_{L^{\rho}(X)} \leq\left\|T^{\prime}\right\|^{p^{\prime} / p}\|f\|_{L^{p}(X)}^{p^{\prime} q / q^{\prime} p}
$$

so that $\|f\| \leq 1 \Rightarrow\|S f\| \leq\left\|T^{\prime}\right\|^{p^{\prime} / p}$. Hence there exists $\alpha \neq 0, \alpha \geq 0, \alpha \in$ $L^{p}(X)$ with norm less than or equal to 1 and $S(\alpha) \leq 2\left\|T^{\prime}\right\|^{p^{\prime} / p} \alpha$. The proof of theorem 1 is finished by taking

$$
u_{0}=\alpha v^{-1 / p}, v_{0}=T\left(u_{0}\right) /\|T\|, u_{1}=\left(T\left(u_{0}\right) /\|T\|\right)^{q / q^{\prime}} u
$$

and $v_{1}=u_{0}^{p / p^{\prime}} v$.

Theorems 2 and 3 will be established once we prove the sufficiency of the conditions, since the necessity follows immediately from theorem 1. To prove theorem 2 take $f \in L^{p}(X, v), g \in L^{q^{\prime}}\left(Y, u^{-q^{\prime} / q}\right)$ and use Holder's inequality with $r, p$ and $q^{\prime}$, where $\frac{1}{r}=\frac{1}{q}-\frac{1}{p}$ to obtain 


$$
\begin{aligned}
\left|\int_{X} \int_{Y} k(x, y) f(x) g(y) d x d y\right| & \leq\left(\int_{X} \int_{Y} k(x, y) u_{1}(y) u_{0}(x) d x d y\right)^{1 / r} \\
& \left(\int_{X} \int_{Y} k(x, y) u_{3}(y) u_{0}(x)^{-p / p^{\prime}}|f(x)|^{p} d x d y\right)^{1 / p} \\
& \cdot\left(\int_{X} \int_{Y} k(x, y) u_{0}(x) u_{1}(y)^{-q^{\prime} / q}|g(y)|^{q^{\prime}} d x d y\right)^{1 / q^{\prime}} .
\end{aligned}
$$

Jsing $K^{*}\left(u_{1}\right) \leq C_{1} v_{1}$ and $\left\|u_{0} v_{1}\right\|_{L^{1}(X)} \leq 1$ the first factor on the right hand side of the above inequality is bounded by $C_{1}^{1 / r}$. Using $K^{*}\left(u_{1}\right) \leq C_{1} v_{1}$ and $v=u_{0}^{-p / p^{\prime}} v_{1}$ we deduce that the second factor is bounded by $C_{1}^{1 / p}\|f\|_{L^{p}(X, v)}$. Finally, using $K\left(u_{0}\right) \leq C_{0} v_{0}$ and $u=v_{0}^{-q / q^{\prime}} u_{1}$ the third factor can be majorated by $C_{0}^{1 / q^{\prime}}\|g\|_{L^{\prime}\left(Y, u^{\prime} q^{\prime} / q\right)}$. Putting these cstimates together we obtain

$$
\left.\left|\int_{X} \int_{Y} k(x, y) f(x) g(y) d x d y\right| \leq C_{0}^{1 / q^{\prime}} C_{1}^{1 / q}\|f\|_{L^{p}(X ; v)}\|g\|_{L\left(Y ; u^{-q^{\prime} / q}\right.}^{q^{\prime}}\right) .
$$

From here the desired result follows.

The same argument applies for $p=q$, that is for theorem 3 , except that in this case $\frac{1}{r}=\frac{1}{q}-\frac{1}{p}=0$, and hence the first factor on the right hand side of (2.2) does not appear. Thus theorem 3 docs not require the condition $\left\|u_{0} v_{1}\right\|_{L^{1}(X)} \leq 1$.

To prove theorem 4 let $f \in L^{p}(X, v)$ and $g \in L^{q^{\prime}}\left(Y, u^{-q^{\prime} / q}\right)$. From $\frac{1}{r}=\frac{1}{q}-\frac{1}{p}$ we deduce $\frac{1}{q^{\prime}}+\frac{1}{p}=\frac{1}{r^{\prime}}$ so that we can apply Holder's inequality with indices $p / r^{\prime}$ and $q^{\prime} / r^{\prime}$ to obtain

$$
\begin{aligned}
& \left|\int_{X} \int_{Y} k(x, y) f(x) g(y) d x d y\right| \\
& \leq\left(\int_{X} \int_{Y} k(x, y) u_{1}(y) u_{0}(x)^{-p / q^{\prime}}|f(x)|^{p / r^{\prime}} d x d y\right)^{r^{\prime} / p} \\
& \quad\left(\int_{X} \int_{Y} k(x, y) u_{0}(x) u_{1}(y)^{-q^{\prime} / p}|g(y)|^{q^{\prime} / r^{\prime}} d x d y\right)^{r^{\prime} / q^{\prime}}=(I) .(I I) .
\end{aligned}
$$

Using Holder's inequality with index $r$, together with $v=u_{0}^{-p / p^{\prime}} v_{1}^{q^{\prime} / p^{\prime}}$ and $K^{*}\left(u_{1}\right) v_{1}^{-1} \in L^{r}\left(v^{-p^{t} / p}\right)$ we obtain

$$
\left.(I) \leq\left(\int_{X}\left[K^{*} u_{1}\right)(x)\right]^{r} v_{1}^{-r}(x) v^{-p^{\prime} / p}(x) d x\right)^{r^{\prime} / r p}
$$




$$
\begin{gathered}
\left(\int_{X}|f(x)|^{p} v_{1}(x)^{r^{\prime}} v(x)^{p^{\prime} r^{t} / p r} u_{0}(x)^{-p r^{\prime} / q^{\prime}} d x\right)^{1 / p} \\
=C_{1}^{r^{\prime} / p}\left(\int_{X}|f(x)|^{p} v(x) d x\right)^{1 / p}
\end{gathered}
$$

Using again Holder's inequality with the same index, together with $u=$ $u_{1} v_{0}^{-p / q^{\prime}}$ and $K\left(u_{0}\right) v_{0}^{-1} \in L^{r}(u)$ we obtain

$$
\begin{gathered}
(I I) \leq\left(\int\left[K\left(u_{0}\right)(y)\right]^{r} v_{0}(y)^{-r} u(y) d y\right)^{r^{\prime} / r q^{\prime}} \\
\cdot\left(\int_{y}|g(y)|^{g^{\prime}} u_{1}(y)^{-q^{\prime} r^{\prime} / p} v_{0}(y)^{r^{\prime}} u(y)^{-r^{\prime} / r} d y\right)^{1 / q^{\prime}} \\
=C_{0}^{r^{\prime} / q^{\prime}}\left(\int_{y}|g(y)|^{q^{\prime}} u(y)^{-q^{\prime} / q} d y\right)^{1 / q^{\prime}}
\end{gathered}
$$

The desired result follows by putting these two estimates together. This finishes the proof of theorem 4.

We now prove theorem 5 .

(A) Sufficiency. For $f \in L^{p}(X, v)$ and $g \in \underline{L}^{\infty}\left(Y, u^{-1}\right)$ we have

$$
\begin{gathered}
\left|\int_{X} \int_{Y} k(x, y) f(x) g(y) d x d y\right| \leq\|g\|_{L^{\infty}\left(Y, u^{-1}\right)} \int_{X} \int_{Y} k(x, y)|f(x)| u(y) d x d y \\
\leq\|g\|_{\underline{L}^{\infty}\left(Y, u^{-1}\right)}\left\{\int_{X}\left(\int_{Y} k(x, y) u(y) d y\right)^{p^{\prime}} v(x)^{-p^{\prime} / p} d x\right\}^{1 / p^{\prime}} \\
\left\{\int_{X}|f(x)|^{p} v(x) d x\right\}^{1 / p} \leq\|g\|_{\underline{L}^{\infty}\left(Y, u^{-1}\right)}\|K\|\|f\|_{L^{p}(X, v)} .
\end{gathered}
$$

Necessity. The boundedness of $K$ implies

$$
\left|\int_{X} \int_{Y} k(x, y) f(x) g(y) d y d x\right| \leq\left\|K^{r}\right\|\|f\|_{L^{p}(X, v)}\|g\|_{L^{\infty}\left(Y, u^{-1}\right)}
$$

for all $f \in L^{p}(X, v), g \in \underline{L}^{\infty}\left(Y, u^{-1}\right)$. With $g \equiv u$ we obtain

$$
\left|\int_{X} f(x)\left(\int_{Y} k(x, y) u(y) d y\right) d x\right| \leq\|K\|\|f\|_{L}(X, v)
$$

for all $f \in L^{p}(X, v)$. Thus the result follows.

(B) The proof is analogous. 


\section{Applications}

Consider the integral transformations

$$
(K f)(x)=\int_{-\infty}^{x} k(x, y) f(y) d y,\left(K^{*} f\right)(x)=\int_{x}^{\infty} k(y, x) f(y) d y
$$

defined on the real line, where $k(x, y)$ is a nonnegative measurable function defined on $\Delta=\left\{(x, y) \in \mathrm{R}^{2}: y<x\right\}$. Given two non-negative measurable functions $u$ and $v$ defined on the real line, we write $(u, v) \in W_{1}(K, p, q), 1 \leq$ $q \leq p<\infty$ if

(3.2) $\quad B_{1}=$

$$
\left\{\int_{-\infty}^{\infty}\left[\left(\int_{y}^{\infty} k(x, y) u(x) d x\right)^{1 / q}\left(\int_{-\infty}^{y} k(y, z) v(z)^{-p^{\prime} / p} d z\right)^{1 / q}\right]^{r} v(y)^{-p^{\prime} / p} d y\right\}^{1 / r}
$$

and $(u, v) \in W_{2}(K, p, q), 1<q \leq p \leq \infty$ if

(3.3) $\quad B_{2}=$

$$
\left\{\int_{-\infty}^{\infty}\left[\left(\int_{y}^{\infty} k(x, y) u(x) d x\right)^{1 / p}\left(\int_{-\infty}^{y} k(y, z) v(z)^{-p^{\prime} / p} d z\right)^{1 / p^{\prime}}\right\}^{r} u(y) d y\right\}^{1 / r}
$$

where $\frac{1}{r}=\frac{1}{q}-\frac{1}{p}$. Observe that in the limiting case $p=q,(3.2)$ and (3.3) become

$$
B=\sup _{y>0}\left(\int_{y}^{\infty} k(x, y) u(x) d x\right)^{1 / p}\left(\int_{-\infty}^{y} k(y, z) v(z)^{-p^{\prime} / p} d z\right)^{1 / p^{\prime}}<+\infty
$$

Proposition 3.1. Let $K$ be the integral transformation defined by (3.1), where $k(x, y) \geq 0$ is nondecreasing in $y$ and nonsncreasing in $x$. If $(u, v) \in$ $W_{j}(K, p, q), 1<q \leq p<\infty, j=1,2$, then

$$
\left(\int_{-\infty}^{\infty}|(K f)(x)|^{q} u(x) d x\right)^{1 / q} \leq C\left(\int_{-\infty}^{\infty}|f(x)|^{p} v(x) d x\right)^{1 / p}
$$


where $C \leq\left(q B_{1}\right)^{r^{\prime} / p}\left(p^{\prime} B_{2}\right)^{r^{\prime} / q^{*}}$.

Proof: Since $t \geq y$ implies $k(x, t) \geq k(x, y)$ the norm of the function

$$
\begin{aligned}
& \varphi(y)= \\
& \left(\int_{y}^{\infty} k(t, y)\left(\int_{t}^{\infty} k(x, t) u(x) d x\right)^{-1 / q^{\prime}} u(t) d t\right)\left(\int_{-\infty}^{y} k(y, z) v(z)^{-p^{\prime} / p} d z\right)^{1 / q^{\prime}}
\end{aligned}
$$

in $L^{r}\left(v^{-p^{\prime} / p}\right)$ is bounded by the norm of the function

$$
\begin{aligned}
& \tilde{\varphi}(y)= \\
& \left(\int_{y}^{\infty} k(t, y)\left(\int_{t}^{\infty} k(x, y) u(x) d x\right)^{-1 / q^{\prime}} u(t) d t\right)\left(\int_{-\infty}^{y} k(y, z) v(z)^{-p^{\prime} / p} d z\right)^{1 / q^{\prime}}
\end{aligned}
$$

in the same space. Integrating by parts we obtain

$$
\|\varphi\|_{L^{r}\left\{v^{\left.-p^{\prime} / p\right)}\right.} \leq q B_{1} .
$$

Taking

$$
\begin{aligned}
& u_{1}(t)=\left(\int_{t}^{\infty} k(x, t) u(x) d x\right)^{-1 / q^{\prime}} u(t), \\
& v_{1}(y)=\left(\int_{-\infty}^{y} k(y, z) v(z)^{-p^{\prime} / p} d z\right)^{-1 / q^{\prime}}
\end{aligned}
$$

the above inequality can be written as $K^{*}\left(u_{1}\right) v_{1}^{-1} \in L^{r}\left(v^{-p^{\prime} / p}\right)$ with norm not exceeding $q B_{1}$.

Since $t \leq y$ implies $k(t, z) \geq k(y, z)$, the norm of the function

$$
\begin{aligned}
& \phi(y)= \\
& \left(\int_{y}^{\infty} k(x, y) u(x) d x\right)^{1 / p}\left(\int_{-\infty}^{y} k(y, t) v(t)^{-p^{\prime} / p}\left(\int_{-\infty}^{t} k(t, z) v(z)^{-p^{\prime} / p} d z\right)^{-1 / p} d t\right)
\end{aligned}
$$

in $L^{r}(u)$ is bounded by the norm of the function

$$
\begin{aligned}
& \tilde{\phi}(y)= \\
& \left(\int_{y}^{\infty} k(x, y) u(x) d x\right)^{1 / p} \int_{-\infty}^{y} k(y, t) v(t)^{-p^{\prime} / p}\left(\int_{-\infty}^{t} k(y, z) v(z)^{-p^{\prime} / p} d z\right)^{-1 / p} d t
\end{aligned}
$$


in the same space. Integrating by parts we obtain

$$
\|\phi\|_{L^{r}(u)} \leq p^{\prime} B_{2} .
$$

Taking

$$
\begin{aligned}
& u_{0}(t)=\left(\int_{-\infty}^{t} k(y, z) v(z)^{-p^{\prime} / p} d z\right)^{-1 / p} v(t)^{-p^{2} / p}, \\
& v_{0}(t)=\left(\int_{t}^{\infty} k(x, t) u(x) d x\right)^{-1 / p}
\end{aligned}
$$

the above inequality can be written as $K\left(u_{0}\right) v_{0}^{-1} \in L^{r}(u)$ with norm not exceeding $p^{\prime} B_{2}$. The proof of proposition 3.1 is finished by applying theorem 4 if $q<p$ and theorem 3 if $q=p$.

Remarks. 1. For the case $q=1,(u, v) \in W_{1}(K, p, 1)$ is an equivalent condition for the boundedness of the operator $K$ defined by (3.1) from $L^{p}(X, v)$ to $L^{1}(Y, u)$. This follows from part (A) of theorem 5 .

2. For the case $p=\infty,(u, v) \in W_{2}(K, \infty, q)$ is an equivalent condition for the boundedness of $K$ from $L^{\infty}(X, v)$ to $L^{q}(Y, u)$. This follows from part (B) of theorem 5 .

3. A result similar to proposition 3.1. can be obtained for $K^{*}$. Details are left to the interested reader.

4. The operators defined by (3.1) have been studied in [2]; the conditions imposed on the weights $u$ and $v$ to obtain weighted inequalities for $K$ are different from those used here.

For the particular case of the Hardy opcrator $T f(x)=\int_{0}^{x} f(y) d y, W_{1}(T, p, q)$ becomes

$$
B_{1}=\left\{\int_{0}^{\infty}\left[\left(\int_{y}^{\infty} u\right)^{1 / q}\left(\int_{0}^{y} v^{-p^{\prime} / p}\right)^{1 / q^{\prime}}\right]^{r} v(y)^{-p^{\prime} / p} d y\right\}^{1 / r}<+\infty,
$$

$W_{2}(T, p, q)$ becomes

$$
B_{2}=\left\{\int_{0}^{\infty}\left[\left(\int_{y}^{\infty} u\right)^{1 / p}\left(\int_{0}^{y} v^{-p^{\prime} / p}\right)^{1 / p^{r}}\right]^{r} u(y) d y\right\}^{1 / r}<+\infty,
$$

and when $p=q$ we have

$$
B=\sup _{y>0}\left(\int_{y}^{\infty} u\right)^{1 / p}\left(\int_{0}^{y} v^{-p^{\prime} / p}\right)^{1 / p^{\prime}}<+\infty .
$$

In this case we shall show that the condition $W_{2}(T, p, q)$ is implied by $W_{1}$ $(T, p, q)$ and that this condition is also necessary for the boundedness of $T$. 
Proposition 3.2. Let $1<q \leq p<\infty$. A necessary and sufficient condition for the Hardy operator $T f(x)=\int_{0}^{x} f(y) d y$ to be bounded from $L^{p}(v)$ to $L^{q}(u)$ with norm $C$ is $(u, v) \in W_{1}(T, p, q)$. Moreover $2^{-p / p^{\prime} q} B_{1} \leq C \leq$ $2^{r^{\prime} / r q^{\prime}} B_{1}\left(p^{\prime}\right)^{1 / q^{\prime}} q^{1 / q}$

Proof: Suffciency. Reducing the interval of integration and using the condition $W_{1}(T, p, q)$ we deduce

$$
\left(\int_{z}^{\infty} u\right)^{1 / q}\left(\int_{0}^{z} v^{-p^{\prime} / p}\right)^{1 / p^{\prime}}\left(\frac{p^{\prime}}{r}\right)^{1 / r} \leq B_{1}
$$

for all $z \in(0, \infty)$. Integrating by parts in (3.5) and using (3.8) we obtain

$$
\begin{aligned}
\left.B_{1}^{r}=\frac{p^{\prime}}{r}\left(\int_{y}^{\infty} u\right)^{r / q}\left(\int_{0}^{y} v^{-p^{\prime} / p}\right)^{r / p^{\prime}}\right]_{0}^{\infty}+ \\
\quad \frac{p^{\prime}}{q} \int_{0}^{\infty}\left(\int_{y}^{\infty} u\right)^{r / p}\left(\int_{0}^{y} v^{-p^{\prime} / p}\right)^{r / p^{\prime}} u(y) d y \geq-B_{1}^{r}+\frac{p^{\prime}}{q} B_{2}^{r} .
\end{aligned}
$$

Hence $B_{2}^{r} \leq 2 B_{1}^{r} \frac{q}{p^{\prime}}$ and the result now follows from proposition 3.1.

Necessity. Since we are assuming that $T$ is bounded from $L^{p}(v)$ to $L^{q}(u)$, we can apply theorem 1 to find $u_{0}, v_{0}, u_{1}, v_{1}$ satisfying

$$
\left\|u_{0} v_{1}\right\|_{L^{\prime}} \leq 1
$$

and

$$
v=u_{0}^{-p / p^{*}} v_{1}, u=v_{0}^{-q / q^{\prime}} u_{1}, T\left(u_{0}\right) \leq C v_{0}, T^{*}\left(u_{1}\right) \leq 2^{p / p^{\prime}} C v_{1} .
$$

Therefore

$$
B_{1}^{r}=\int_{0}^{\infty}\left(\int_{y}^{\infty} v_{0}(t)^{-q / q^{\prime}} u_{1}(t) d t\right)^{r / q}\left(\int_{0}^{y} u_{0}(t) v_{1}(t)^{-p^{\prime} / p} d t\right)^{r / q^{\prime}} v(y)^{-p^{\prime} / p} d y .
$$

From $T u_{0} \leq C v_{0}$ and $T^{*}\left(u_{1}\right) \leq 2^{p / p^{\prime}} C v_{\xi}$ we deduce that $B_{1}^{r}$ is bounded by

$$
\begin{aligned}
2^{r / q^{\prime}} C^{r p^{\prime} / q^{\prime}} & \left\{\int_{0}^{\infty}\left(\int_{0}^{y} u_{0}\right)^{-r / q^{\prime}}\left(\int_{y}^{\infty} u_{1}\right)^{r / q}\right. \\
& \left.\left(\int_{y}^{\infty} u_{1}\right)^{-r p^{\prime} / p q^{\prime}}\left(\int_{0}^{y} u_{0}\right)^{r / q^{\prime}} u_{0}(y) v_{1}(y)^{-p^{\prime} / p} d y\right\} \\
& \leq 2^{r / p^{\prime}} C^{r-1}\left\{\int_{0}^{\infty}\left(\int_{y}^{\infty} u_{1}\right) u_{0}(y) d y\right\} \\
& \leq 2^{(r+p) / p^{\prime}} C^{r} \int_{0}^{\infty} v_{1}(y) u_{0}(y) d y \leq 2^{(r+p) / p^{\prime}} C^{r}
\end{aligned}
$$


where the last inequality is due to (3.9). This finishes the proof of proposition 3.2 .

Remarks. 1. The limiting cases $q=1$ and $p=\infty$ of proposition 3.2 are also true; they can be deduced directly from theorem 5 .

2. There is a similar result for the dual of the Hardy operator, $T^{*} f(x)=$ $\int_{x}^{\infty} f(y) d x$; details are left for the interested reader.

3. Proposition 3.2 can be found in [6] for the case $p=q$ and in [5] for $q<p$. We feel that our proof is easicr than that given in [5], page 45 .

To end this section we use theorems 2 and 3 to find particular weights for the Laplace transform $\mathcal{L}$ and the Riemann-Liouville operator $T_{\alpha}, \alpha>0$.

Corollary 3.3. Let $1<p<\infty$ and $a>-1$. Then

$$
\left\{\int_{0}^{\infty}|(\mathcal{L} f)(x)|^{p} x^{a} d x\right\}^{1 / p} \leq C\left\{\int_{0}^{\infty}|f(x)|^{p} x^{-a+p-2} d x\right\}^{1 / p}
$$

and $C \leq \Gamma(\beta+1)^{1 / p^{\prime}} \Gamma^{\prime}\left(a-\beta \frac{p}{p^{\prime}}+2-p\right)^{1 / p}$ for all $-1<\beta<\frac{p^{\prime}}{p} a+\frac{p^{\prime}}{p}-1$.

Proof: Since $a>-1$ we can choose $\beta$ such that $-1<\beta<\frac{p^{\prime}}{p} \alpha+\frac{p^{\prime}}{p}-1$. Let $u_{0}(x)=x^{\beta}$ and $v_{1}(x)=x^{-a+p-2+\beta \frac{p}{p}}$ so that $u_{0}^{-p / p^{\prime}} v_{1}=x^{-a+p-2}$. We have $\left(\mathcal{L} u_{0}\right)(x)=\Gamma(\beta+1) / x^{\beta+1}$. Let $v_{0}(x)=x^{-\{\beta+1)}$ and $u_{1}(x)=x^{a-(\beta+1) \frac{p}{\beta^{\prime}}}$ so that $v_{0}^{-p / p^{\prime}} u_{1}=x^{a}$. Moreover $\left(\mathcal{L} u_{1}\right)(x)=\Gamma\left(\alpha-(\beta+1) \frac{p}{p^{\prime}}+1\right) / x^{a-\{\beta+1) \frac{\beta}{p}+1}=$ $\Gamma\left(a-(\beta+1) \frac{p}{p^{\prime}}+1\right) v_{1}(x)$. Hence the result follows by theorem 3 .

Corollary 3.4. Let $\alpha>0,1<p<\infty$ and $b<p-1$. Then

$$
\left\{\int_{0}^{\infty}\left|\left(T_{\alpha} f\right)(x)\right|^{p} x^{b-\alpha p} d x\right\}^{1 / p} \leq C\left\{\int_{0}^{\infty}|f(x)|^{p} x^{b} d x\right\}^{1 / p}
$$

and $C \leq B(\alpha, \beta+1)^{1 / p^{\prime}} B\left(\alpha,-b-\frac{p}{p^{\prime}} \beta\right)^{1 / p} \Gamma(\alpha)^{-1}$ for all $-1<\beta<-\frac{p^{\prime}}{p} b$, where $B$ is the beta function, $B(s, r)=\int_{0}^{1}(1-x)^{s-1} x^{r-1} d x$.

Proof: Since $b<p-I$ we can choose $\beta$ so that $-1<\beta<-\frac{p^{\prime}}{p}$. Let $u_{0}(x)=$ $x^{\beta}$ and $v_{1}(x)=x^{b+\beta \frac{p}{p^{\prime}}}$ so that $u_{0}^{-p / p^{\prime}} v_{1}=x^{b}$. A simple calculation shows $\left(T_{\alpha} u_{0}\right)(x)=x^{\alpha+\beta} B(\alpha, \beta+1) / \Gamma(\alpha)$. Let $v_{0}(x)=x^{\alpha+\beta}$ and $u_{1}=x^{b-\alpha p+\frac{p}{p^{\prime}}(\alpha+\beta)}$ so that $v^{-p / p^{\prime}} u_{1}=x^{b-\alpha p}$. Again a calculation shows

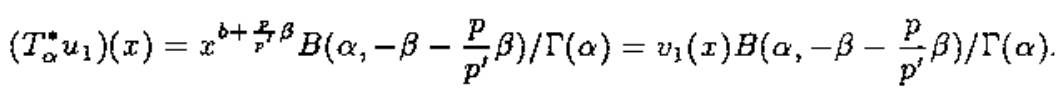

The result follows from theorem 3 . 
Corollary 3.5. Let $1 \leq q<p<\infty$ and let a be such that $(q / p)-\left(q / q^{\prime}\right)<$ $a<1-\left(q / q^{t}\right)$ (if $\left.q=1, \frac{1}{p}<a<1\right)$. Then

$$
\left(\int_{0}^{\infty}|\mathcal{L} f(x)|^{q} x^{-a} d x\right)^{1 / q} \leq C\left(\int_{0}^{\infty}|f(x)|^{p}\left(1+x^{2}\right)^{p-1} d x\right)^{1 / p} .
$$

Proof: Suppose $1<q<p<\infty$. Choose $\beta$ so that $\beta\left(\frac{p}{p^{\prime}}-\frac{q}{q^{t}}\right)=a+\frac{q}{q^{t}}-1$. The conditions on $a$ imply $-\frac{1}{p}<\beta<0$. Let $u_{0}(x)=x^{\beta}\left(1+x^{2}\right)^{-1}$ and $v_{1}(x)=x^{\beta \frac{p}{p}}$ so that $u_{0}^{-p / p^{\prime}} v_{1}=\left(1+x^{2}\right)^{p-1}$. Observe that

$$
\int_{0}^{\infty} u_{0} v_{1}=\int_{0}^{\infty} x^{\beta p}\left(1+x^{2}\right)^{-1} d x<\infty
$$

since $\beta p+1>0$ and $\beta p-1<0$. Now

$$
\left(\mathcal{L} u_{0}\right)(x)=\int_{0}^{\infty} e^{-x t} t^{\beta}\left(1+t^{2}\right)^{-1} d t \leq \int_{0}^{\infty} e^{-x t} t^{\beta} d t=x^{-\beta-1} \Gamma(\beta+1) .
$$

Let $v_{0}(x)=x^{-\beta-1}$ and $u_{1}(x)=x^{-a-(\beta+1) \frac{q}{q}}$ so that $v_{0}^{-q / q^{+}} u_{1}=x^{-a}$. Moreover $\left(\mathcal{L} u_{1}\right)(x)=v_{1}(x) \Gamma\left(-\beta \frac{p}{p^{\prime}}\right)$. The result follows by applying theorem 2 .

The case $q=1$ follows from part $(A)$ of theorem 5 .

Corollary 3.6. Let $1 \leq q<p<\infty$ and let a be such that $-1+\frac{q}{p}-\alpha q<$ $a<-\alpha q$ where $\alpha>0$. Then

$$
\left.\left.\left(\int_{0}^{\infty} \mid T_{\alpha} f(x)\right)\right|^{q} x^{a} d x\right)^{1 / q} \leq C\left(\int_{0}^{\infty}|f(x)|^{p} e^{x b} d x\right)^{1 / p}, b>0
$$

Proof: Suppose $1<q<p<\infty$. Choose $\beta$ such that $\beta\left(\frac{p}{p^{\prime}}-\frac{q}{q^{\prime}}\right)=\alpha+q \alpha$. The conditions on $a$ imply $-\frac{1}{p}<\beta<0$. Let $u_{0}(x)=x^{\beta} e^{\frac{z^{\prime}}{p} x}$ and $v_{1}(x)=x^{\beta \frac{p}{p^{\prime}}}$ so that $u_{0}^{-p / p^{\prime}} v_{1}=e^{x b}$. Observe that

$$
\int_{0}^{\infty} u_{0} v_{1}=\int_{0}^{\infty} x^{p \beta} e^{\frac{p^{\prime}}{p} x} d x=C \Gamma(p \beta+1)<\infty
$$

since $p \beta+1>0$. Now

$$
\begin{aligned}
\left(T_{\alpha} u_{0}\right)(x) & =\frac{1}{\Gamma(\alpha)} \int_{0}^{x} \frac{t^{\beta} e^{-\frac{p^{\prime}}{p} 1 b}}{(x-t)^{1-\alpha}} d t \leq \frac{1}{\Gamma(\alpha)} \int_{0}^{x} \frac{t^{\beta}}{(x-t)^{1-\alpha}} d t \\
& =x^{\beta+\alpha} B(\alpha, \beta+1) / \Gamma(\alpha) .
\end{aligned}
$$

Let $v_{0}(x)=x^{\beta+\alpha}$ and $u_{1}(x)=x^{\alpha+\frac{s}{q}(\beta+\alpha)}$ so that $v_{0}^{-q / q^{\prime}} u_{1}=x^{a}$. Moreover $\left(T_{\alpha}^{*} u_{1}\right)(x)=v_{1}(x) B\left(\alpha,-a-\frac{q}{q^{t}}(\beta+\alpha)-\alpha\right) / \Gamma(\alpha)$ and the results follow from theorem 2 .

The case $q=1$ follows from part A of theorem 5 . 


\section{References}

[1] Gagliardo, E., On integral transformations with positive kernel, Proc. Amer, Math. Soc. 16 (1965), 429-434.

[2] HEINIG, H.P., Weighted norm inequalities for certain integral operators II, Proc. Amer. Math. Soc. 95 (1985), 387-395.

[3] Hernández, E., Factorization and extrapolation of pairs of weights, Studia. Math. 95 (1989), 179-193.

[4] Jones, P., Factorization of Ap weights, Ann. of Math. 111 (1980), $511-530$.

[5] MAZ'YA, V.G., "Sobolev spaces," Springer-Verlag, 1988.

[6] Muckenhoupt, B., Hardy's inequality with weights, Studia Math. 44 (1972), 31-38.

[7] MuCKenhoupt, B., Weighted norm inequalities for classical operators, Proc. Sympos. Pure Math. 35, 1 (1979), 69-83.

[8] RUIZ, F.J. AND TORREA, J.L., Factorization and extrapolation of pairs of weights in two different measure spaces, Math. Nachr. (to appeas). 